\title{
Chapter 18 \\ Building Integrated Explanatory Models of Complex Biological Phenomena: From Mill's Methods to a Causal Mosaic
}

\begin{abstract}
Alan Love
Abstract Mill's methods involve two idealizations ("one cause, one effect" and "no mixing of effects"), but causal relationships in biology usually exhibit a plurality of causes and intermixture of effects. Building explanatory models to capture these relations remains a challenge because similar idealizations occur in contemporary causal reasoning (e.g., difference making). The problem is poignant for formulating integrated models of different types of causes, such as combining physical and genetic causes to understand their joint contribution to anatomical structures in embryogenesis. Standardized periodizations can help in formulating integrated explanatory models within developmental biology that are causal mosaics of reasoning from difference making and production (mechanism) accounts. A consequence of this strategy is a tradeoff between models that yield causal generalizations of wide scope and models that locally integrate different types of causes to comprehensively explain complex phenomena.
\end{abstract}

Keywords Developmental biology - Difference making • Explanation

- Genetics • Idealization • Integration • Mechanisms • Physics

\subsection{The Challenge of Integration}

Building explanatory models of complex phenomena is a central challenge in diverse sciences. The problem is poignant when the aim is to offer an integrated account of how different causes make contributions to complex biological phenomena (Mitchell 2003). How are different types of causes "combined" in scientific practice to explain how an effect results from their joint operation? This difficulty

The original version of this chapter was revised.

An erratum to this chapter can be found at https://doi.org/10.1007/978-3-319-53730-6_29

A. Love $(\square)$

Department of Philosophy, Minnesota Center for Philosophy of Science, University of Minnesota, 831 Heller Hall, 271 19th Ave. S, Minneapolis, MN, 55455, USA

e-mail: aclove@umn.edu 
is manifested in questions about how to combine physical and genetic causes of embryogenesis (Miller and Davidson 2013). Genetic explanations appeal to changes in the expression of genes and interactions among their RNA and protein products to causally explain how processes of differentiation, pattern formation, growth, and morphogenesis produce anatomical structures, such as a heart or limb. Physical explanations appeal to mechanical forces resulting from the geometrical arrangements of soft condensed materials within the embryo to causally explain the same effects. There is no controversy about whether both are involved: "both the physics and biochemical signaling pathways of the embryo contribute to the form of the organism" (Von Dassow et al. 2010,1). The issue is how to combine the different types of causes to understand their joint contribution to the effect of organismal form: "it is necessary to combine molecular insights (genes and morphogens) with knowledge of physical processes (transport, deformation and flow) generated by growing tissues" (Savin et al. 2011, 57).

In this paper I demonstrate that representations of time are an underappreciated dimension of building integrated explanatory models. I begin with a historical source-Mill's methods-where two typical idealizations are clearly articulated: "one cause, one effect" and "no intermixture of effects." These two idealizations knowingly depart from the situation found in complex biological phenomena: a plurality of causes and intermixture of effects. The significance of attending to this historical moment is that idealizations akin to "one cause, one effect" and "no intermixture of effects" are embedded in contemporary causal reasoning, such as difference making (Illari and Russo 2014), and therefore germane when attempting to combine physics and genetics in explanatory models. Another idealization in Mill's methods - no representation of time apart from an ordinal relation of causes preceding their effects - is shared by causal reasoning based on difference making, but not by production accounts (e.g., mechanisms). However, even though descriptions of mechanisms can incorporate representations of temporal duration between difference makers, the forms of integration common to mechanistic explanation are ill suited to combining genetic and physical causes.

I argue that standardized periodizations in developmental biology can serve as a framework to combine genetic and physical causes. These permit the linkage of difference makers into chains of productive continuity-a standard criterion on mechanistic descriptions (Craver and Darden 2013). Relations of systematic dependence between genetic and physical factors can be keyed to different aspects of a sequence during which the mediation of cause and effect occurs. These representations of time are not tied to a particular mechanistic description, but are localized to a model organism and applicable to many different kinds of mechanisms. The result is a strategy for building integrated explanatory models of complex biological phenomena that does not privilege one conception of causality; instead, it derives from a "causal mosaic" that coordinates different types of causal reasoning for the purpose of explanation (Illari and Russo 2014). In conclusion, I note a counterintuitive result of this strategy: it narrows the scope of generalizations ascertained for the explanatory models in isolation. This suggests a tradeoff between models with causal generalizations of wide scope and models that integrate different types of causes to more comprehensively explain complex phenomena. 


\subsection{Mill's Methods and Idealization}

After his discussion of the four methods of observation and experimental inquiry (Agreement, Difference, Residues, and Concomitant Variations), John Stuart Mill raised a concern about the applicability of these methods to complex causal relationships.

It has been necessary to suppose... for the sake of simplification, that this analytical operation is encumbered by no other difficulties than what are essentially inherent in its nature; and to represent to ourselves, therefore, every effect, on the one hand as connected exclusively with a single cause, and on the other hand as incapable of being mixed and confounded with another coexistent effect (Mill 2006, 434).

The simplifying suppositions are idealizations: reasoning strategies that purposefully depart from features known to be present in nature to achieve prediction, explanation, or control (Weisberg 2007). Mill was explicit that "one cause, one effect" and "no mixing of effects" departed from known features of causal relationships and realized they play the role of idealizations in reasoning about the causes of complex phenomena.

It is not true that the same phenomenon is always produced by the same cause: the effect $a$ may sometimes arise from A, sometimes from B. ... The effects of different causes are often not dissimilar, but homogeneous, and marked out by no assignable boundaries from one another: A and B may produce not $a$ and $b$, but different portions of an effect $a$ (Mill 2006, 434).

The first circumstance is "the plurality of causes": more than one cause may produce an effect. The second is "the intermixture of effects": two or more causes may combine to produce an effect. These circumstances are elided in Mill's methods because "one cause, one effect" and "no mixing of causes" purposefully depart from features known to be present in causal relationships.

For Mill, the "one cause, one effect" idealization is more innocent because the plurality of causes primarily handicaps the Method of Agreement. The Method of Difference is unaffected since the negative instance of removing a factor facilitates distinguishing among contributing causes. The intermixture of effects is a thornier problem:

[It] is exemplified by the joint operation of different forces in mechanics, the separate effects of all the causes continue to be produced, but are compounded with one another, and disappear in one total. ... [Effects] are intermingled with, and disguised by, the homogeneous and closely allied effects of other causes $(440,443)$.

Mill addressed this problem with the composition of causes: "if we happen to know what would be the effect of each cause when acting separately from the other, we are often able to arrive deductively, or a priori, at a correct prediction of what will arise from their conjunct agency" (370). When causes compose-and there is a scientific theory of how these causes jointly operate-then one can assign a relative contribution to the effect of different causes even when the intermixture of effects obtains. Mill emphasized that this ability to arrive deductively at a correct prediction of how causes compose is a mark of scientific maturity, distinguishing mechanics from chemistry and physiology. 
Mill's recommendation was not to reduce chemistry or biology to the operation of physical laws but to find laws that will elevate these immature sciences to the deductive mode (i.e., where they have a theory of how chemical or biological causes jointly operate). This would then permit an a priori demonstration of the composition of causes. An intermixture of effects cannot be teased apart by observation or experiment. The joint operation of causes producing the intermixture of effects cannot be disentangled through the Method of Difference: the negative instance of removing the presence of a cause is not possible. Only the deductive method will suffice.

Mill's claim that the deductive method alone can disentangle the relative causal contributions to complex phenomena sets a specific agenda for biological inquiry: "find the law of an effect... from the laws of different tendencies of which it is a joint result" (454). Although Mill was optimistic, this is not good news to the modern philosopher. If there are laws in biology, they are nothing like what Mill envisioned and unlike those found in Newtonian mechanics (Mitchell 2000). And biological phenomena exemplify the intermixture of effects.

In the cases, unfortunately very numerous and important, in which the causes do not suffer
themselves to be separated and observed apart, there is much difficulty in laying down
with due certainty the inductive foundation necessary to support the deductive method.
This difficulty is most of all conspicuous in the case of physiological phenomena; it being
seldom possible to separate the different agencies which collectively compose an organized
body (Mill 2006, 456).

We have little to no expectation that laws of life will be discovered and thereby "upgrade" biology to a deductive science in accord with Mill's vision.

A more recent discussion claims that apportioning causal responsibility (i.e., how much a cause contributes to an effect) is impossible for developmental phenomena because there is no common currency for measuring the contributions (Sober 1988). Sober distinguishes questions of relative contribution from questions of whether a cause makes a difference. Although there are sciences where questions of relative contribution can be addressed (e.g., mechanics), only difference making questions can be asked of ontogeny. Careful experimental methodology can answer whether one type of difference maker accounts for more of the variation in an effect variable for a particular population (e.g., vary the genetic constitution while growing a plant in identical environments or vice versa), but the measure of interaction between variables says only that there is a joint effect of the causes, not how they jointly bring about their effect. This paints a bleak picture for building integrated explanatory models of developmental phenomena.

\subsection{Integrating Genetics and Physics in Explanations of Development}

Little effort is required to translate Mill's discussion of idealizations into the context of contemporary biology. Although there have been major advances in our ability to experimentally dissect biological systems, it is still "seldom possible to 
separate the different agencies which collectively compose an organized body." This inability to distinguish causal contributions makes it difficult to understand how combinations of causes generate complex effects in processes like embryogenesis. If the aim is to build integrated explanatory models for the morphological outcomes of embryogenesis that include both genetics (the presence, absence, or change in frequency of RNA molecules or proteins) and physics (stretching, contraction, compression, fluid shear stress), then the plurality of causes and intermixture of effects must be addressed. Given that some developmental biologists embrace this aim explicitly, ${ }^{1}$ solutions to the difficulty need to be identified.

Importantly, researchers are able to distinguish the contributions of some causes from others (at least to some degree) because the processes producing the effects are extended through time. The fact that there are at least five different ways to form tubes during ontogeny can be teased apart because these processes do not occur instantaneously. The Method of Agreement or Difference can be utilized with less ambiguity when applied at different temporal junctures. This increases their power to discriminate different causal contributions in complex situations and displays another idealization in Mill's methods: the mediating process between cause and effect is treated as irrelevant. In general, this is a characteristic of difference making or "dependence" causal models: "dependence concerns a relation between cause and effect, without concern about what happens in between cause and effect, while production concerns the linking between cause and effect" (Illari and Russo 2014, 252). The pathway from cause to effect is critical for distinguishing situations with the plurality of causes because there is no reason to expect contributing causes to initiate simultaneously. Combinations of causal contributions can be conceptualized not only as "this and that," but also as "this then that."

For the intermixture of effects, the introduction of temporal duration can play a similar role in distinguishing the contributions of different causes to complex outcomes. Consider again Mill's description: "the separate effects of all the causes continue to be produced, but are compounded with one another, and disappear in one total." This disappearance is not total when we incorporate temporality. The separate contributions of causes can be isolated in a sequence where they are compounded into a final effect. An example of this is observable in the origin of aortic arch asymmetry (Yashiro et al. 2007; Fig. 18.1). Although the final morphological outcome is the result of both gene expression and physical dynamics, the organization of these causal factors within a representation of developmental time permits a dissection of how contributions are made. Gene expression at an earlier time makes a difference in the structure of the outflow tract, which leads to a differential distribution of blood flow. This then makes a difference in the regulation of genes on each side, which leads to an asymmetry in aortic arch structure. While this type of explanatory model does not permit a quantitative evaluation of the relative contribution of each type of cause, it does illuminate how types of causes "are compounded with one another, and disappear in one total" and how effects "are

\footnotetext{
${ }^{1}$ See above, Sect. 18.1, in the quotation from Savin et al. (2011).
} 
Fig. 18.1 An explanatory model of the origin of an asymmetric (left-sided) aortic arch with both genetic and physical causes organized into productive continuity by a periodization (Yashiro et al. 2007). Abbreviations: Nodal, Pitx2, PDGFR, VEGFR2 (gene names); OFT outflow tract; $A S$ aortic sac; $B A A$ branchial arch artery (Reprinted by permission from Macmillan Publishers Ltd: Nature (Vol. 450, 287), copyright (2007))

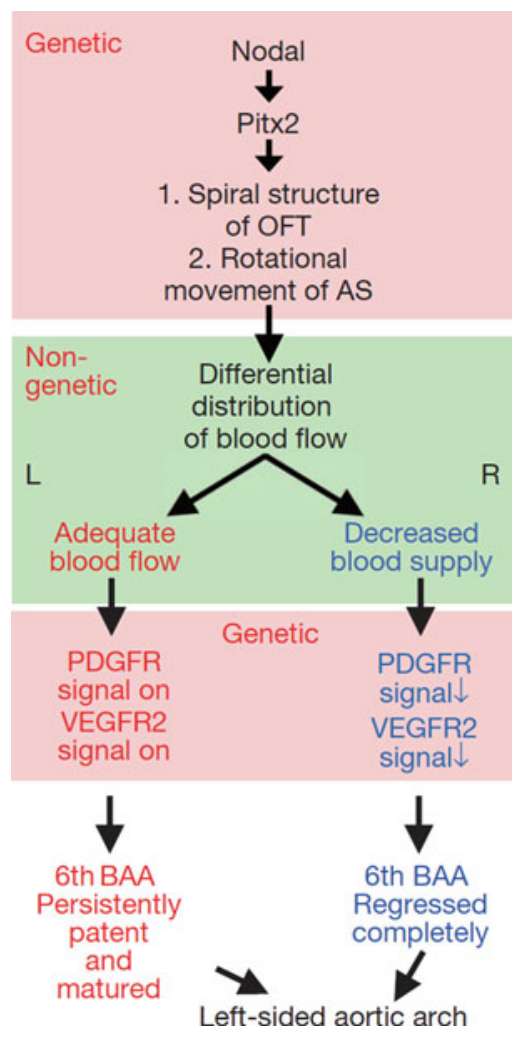

intermingled with, and disguised by, the homogeneous and closely allied effects of other causes." It also aligns directly with the reasoning of developmental biologists: "an increasing number of examples point to the existence of a reciprocal interplay between expression of some developmental genes and the mechanical forces that are associated with morphogenetic movements or with hydrodynamic flows during development" (Brouzés and Farge 2004, 372). ${ }^{2}$

Given the role that representations of time play in these integrated explanatory models, a natural strategy is to adopt a production-oriented causal account, such as mechanisms (Darden 2006). Mechanistic explanations involve decomposing systems into their constituent parts, localizing their characteristic activities, and articulating how they are organized to produce a particular effect. This last element-organization - is crucial to building explanatory models of how an effect is produced by genetic and physical causes during development. However, there are reasons to worry that a mechanisms approach will not solve the problem of finding a suitable model type for constructing integrated explanations.

\footnotetext{
${ }^{2}$ Notice that this rules out interpreting the physics as being in a relationship of realization to the genetics. The relationship is understood as causal and tokens of either type can initiate a chain of interaction.
} 
Craver and Darden (2013, ch. 10) describe three ways that integration can occur in mechanistic explanation. The first is "simple mechanistic integration" where different fields (e.g., molecular biologists and biochemists) are working at the same level or spatial scale of a mechanism, such as the role of RNAs in protein synthesis. Molecular biologists, focusing on an earlier time in protein synthesis, elucidated how messenger RNAs act as a template to guide the incorporation of amino acids via transfer RNAs into a polypeptide that corresponds with the triplet codon derived from a DNA sequence. Biochemists, focusing on a later time, elucidated how individual amino acids that were attached to transfer RNAs bonded to one another to form a stable polypeptide chain. Although different methods and experimental systems were required to ascertain how these aspects of the mechanism operate, eventually they were integrated into a single mechanistic description.

The second form of mechanistic integration is "interlevel integration," which involves exploring mechanisms at different spatial scales or hierarchical levels understood as part-whole relations. For example, to comprehend mechanisms of learning and memory, component mechanisms at the level of the organization of the hippocampus need to be integrated with component mechanisms for how long-term potentiation occurs in individual neurons. These, in turn, need to be integrated with component mechanisms for how specific protein receptors are activated by signaling molecules. Integrating these nested, component mechanisms yields a more robust explanatory model of the mechanisms of learning and memory.

The third form of mechanistic integration is "sequential intertemporal integration." One example is different research communities investigating distinct steps of a complex mechanism where the steps are temporally disjointed, sometimes across generations. The mechanism of heredity involves a complex series of events, such as gene replication, chromosomal rearrangement, and gene expression, each of which contain different relevant component mechanisms (e.g., meiosis or transcription factor binding). Although interlevel integration helps to dissect various steps in this series, one also needs intralevel integration in order to bridge temporal gaps and purchase productive continuity for the mechanistic description. Sequential intertemporal integration requires combining these other two modes of integration across substantial spans of time; otherwise, there are simply black boxes between various steps in the series.

While integrated explanatory models exhibiting these three types can be found in biological practice, it is unclear whether any of them is suitable for building integrated explanatory models of genetic and physical causes operating jointly during development. In light of the plurality of causes and intermixture of effects (e.g., different ways to form a tube or the origin of aortic arch asymmetry), we can see that simple mechanistic integration will be inappropriate because the causal dynamics occurring through the temporal duration are interlevel (e.g., gene expression and fluid flow). Interlevel integration is also inadequate because the mode of explanatory reasoning does not involve decomposing the genetic and physical causal elements into sub-mechanisms before integrating them. This implies that sequential intertemporal integration, which combines features of the other two 
types of mechanistic integration across substantial temporal gaps, is not applicable either. None of these types of mechanistic integration are suitable for combining genetics and physics in explanations of development, even though the causal reasoning associated with mechanisms (i.e., production) foregrounds the temporal dimension that is crucial in overcoming idealizations inherent to difference-making approaches.

That a mechanisms approach to building integrated explanatory models of genetic and physical factors fails is unfortunate; how it fails can lead us in the direction of a solution. First, the reciprocal interaction between genetic and physical causes does not conform to the expectation that mechanism descriptions "bottomout" in lowest level activities of molecular entities (Darden 2006). The interlevel nature of the causal dynamics between genetic and physical factors runs counter to this expectation. Second, the reciprocal interaction between genetic and physical causes does not require stable, compositional organization, which is a key criterion for mechanisms (Darden and Craver 2013). The productive continuity of a sequence of genetic and physical difference makers can be maintained despite changes in the number and types of elements that compose genetic or physical factors in a mechanism. Although compositional differences can alter relationships of physical causation (fluid flow or tension), these relationships do not require the specificity of genetic interaction predominant in most mechanistic explanations from molecular biology (Waters 2007).

\subsection{Periodizations and a Causal Mosaic}

We have a dilemma. If the aim is to offer an integrated account of how different genetic and physical causes make joint contributions to complex biological phenomena during development, then we must confront the plurality of causes and intermixture of effects directly. Difference-making models of causation in the lineage of Mill's methods are highly relevant to experimental reasoning about causes in developmental biology (Waters 2007), but they ignore the productive continuity that illuminates how complex outcomes emerge in biological systems. Therefore, we need a causal model that incorporates representations of time to tease apart causal combinations. A mechanisms approach meets this desideratum through its emphasis on organization. However, the modes of integration available for mechanisms are ill suited for trying to understand the joint contribution of genetics and physics to the effect of organismal form. Thus, there is a necessary role for both types of causal models but neither is sufficient to the task of explanatory integration.

We can escape this dilemma by focusing on what kinds of representations of time are used in scientific practice. Temporal duration for the mechanisms approach is always time "in" the mechanism. In contrast, most developmental explanations use a standardized representation of time - a periodization - that is measured and 
calibrated apart from specific mechanisms. The most ubiquitous of these in developmental biology are normal stages (Hopwood 2005). Stages facilitate the study of different kinds of mechanisms, with different characteristic rates and durations for their stages, within a common framework for a model organism (e.g., Drosophila). They also permit the study of conserved molecular genetic mechanisms in different species because the mechanism description is not anchored to the periodization of the model organism. Let us consider an example of explanatory modeling with genetics and physics that uses a standardized periodization.

Experiments have shown that gene expression is initiated by mechanical deformations of tissue structures in the developing Drosophila embryo (Farge 2003). As germ-band extension occurs during early ontogeny, ${ }^{3}$ the associated physical motions of morphogenesis induce expression of the gene Twist in the anterior foregut and stomodeal primordium (precursor cells of the mouth). Although the protein Dorsal regulates Twist, three experiments showed that Twist is also controlled by mechanical movements: (a) a transient lateral deformation induces the ectopic expression of Twist in the dorsal region of the embryo independent of Dorsal expression; (b) in mutant Drosophila where mechanical movements are blocked, normal expression of Twist in foregut and stomodeal primordium fails to occur, and artificial compression can rescue the mutant phenotype; and, (c) the laser ablation of cells in the dorsal region, which reduces mechanical compression, leads to decreased expression of Twist in the stomodeal primordium. In the absence of the protein Dorsal, these mechanical movements permitted an accumulation of the regulatory protein Armadillo, which subsequently translocates into the nucleus and-in association with other proteins-activates Twist.

In this account of how aspects of the gut form during development, a standard periodization for Drosophila frames the description of causal interactions between physical and genetic factors. The pertinent events (e.g., mesoderm invagination or germ-band extension) correspond to the formal structure of official normal stages, with gastrulation extending through this time: Stage 6 (mesoderm invagination), Stage 7 (early germ-band extension/endodermal anterior midgut invagination), and Stage 10 (stomodeal foregut invagination). These stages, which are calibrated independently of the mechanism of gut formation, permit the linkage of difference makers into chains of productive continuity. ${ }^{4}$ The difference makers are not ranked according to how much variation in the effect variable they account for, but are combined in a periodization, which orders different kinds of causal factors that contribute to an effect. Researchers capture the dependency relations between genetic and physical factors by mapping them onto a temporal sequence during which the mediation of causes and effect occurs. The same set of stages can

\footnotetext{
${ }^{3}$ The germ-band is a coordinated group of cells that develops into the segmented trunk of the embryo. During embryogenesis it extends along the anterior-posterior axis and narrows along the dorsal-ventral axis.

${ }^{4}$ In principle, these difference makers could be linked to stages in the operation of a mechanism. Significantly, in practice, they are not; standardized periodizations underlie the causal reasoning.
} 
accomplish a similar integration with respect to different sets of causes pertinent to other developmental processes. We do not get a composition of causes from this strategy, and therefore no overall sum of relative contributions, but we do achieve an understanding of how multiple causes combine to yield "a correct prediction of what will arise from their conjunct agency." In other words, the strategy explicitly addresses the intermixture of effects.

An advocate of mechanistic explanation might object that one can understand this entire sequence as an "overarching" mechanism that incorporates both physical and biochemical interactions. The objection lacks merit from the perspective of scientific practice. Biologists explicitly distinguish molecular and physical mechanisms, rather than blending them, because they differ in their potential for generalization.

Molecular signaling pathways that control branching morphogenesis appear to be conserved across organs and species. However, despite this molecular homology, recent advances in cell lineage analysis and real-time imaging have uncovered surprising differences in the [physical] mechanisms that build these diverse tissues (Varner and Nelson 2014, 2750).

Generating integrated explanatory models is not equivalent to describing an overarching physico-genetic mechanism because the latter clashes with explanatory practices of generalization prevalent in developmental biology (see below, Sect. 18.5).

Standardized periodizations can act as an epistemological template for combining genetic and physical difference makers from one type of causal reasoning with the productive continuity through time of another: mechanistic production. This instantiates a strategy for building integrated explanatory models of complex biological phenomena that does not privilege a particular conception of causation, but instead combines different accounts into a "causal mosaic" to better comprehend the complexity (Illari and Russo 2014). Biologists do not move from differencemaking accounts to production accounts; they move back and forth between both of them to capture distinct aspects of the complex causal structure of the system.

\subsection{Consequences and Conclusion}

One consequence of this strategy for combining genetic and physical causation in explanations of embryogenesis is a narrowing of the scope of resulting generalizations. The result is counterintuitive: to generate a more integrated explanation for two or more modes of causation, we must give up some of the explanatory generality secured for the modes of causation treated separately. This is a significant loss because many molecular genetic mechanisms are conserved and license general inferences across phylogenetically disparate taxa (e.g., fruit flies and humans). Two reasons account for this loss. First, the periodizations used to assign responsibility to genetic and physical difference makers in chains of productive continuity are specific to the model organisms used in experimentation (e.g., fruit flies). Second, "there are no one-to-one correspondence principles between gene functions and the mechanical events that they affect" (Miller and Davidson 2013, 741). This 
suggests an explanatory tradeoff between specificity of integration in an explanatory model and scope of generalization in non-integrated explanatory models (e.g., molecular genetic causes only). Some researchers clearly value the former (Miller and Davidson 2013), but the latter is part of the rationale for the widespread use of model organisms in developmental biology.

It is worth noting that this consequence clashes with Mill's claim that the deductive method is required to address the intermixture of effects. Mill's approach would secure generality because his solution involved discovering general laws that govern the causes. From this we would be able to calculate the joint effect, a priori, of any combination of genetic and physical causes. However, since such general laws are not available and unlikely to exist, we are either perpetually frustrated in dealing with the intermixture of effects or must adopt an alternative strategy, such as building locally integrated explanatory models using standardized periodizations. This alternative addresses the problem, though at the cost of generality. The causal mosaic strategy of building models that display how genetic and physical difference makers jointly bring about complex outcomes in development is not the only possible strategy for explanatory integration in biological science. Within a mechanisms approach, there are (at least) three different routes to securing mechanistic integration that show how combinations of causes produce an effect. The preceding analysis demonstrates that identifying suitable forms for integrated explanatory models of complex phenomena requires close attention to the details of scientific practice. That was how we identified standardized periodizations as coordinators of different kinds of causal reasoning (difference-making and production), which facilitated integrating genetic and physical factors to understand "what will arise from their conjunct agency."

Since integrated explanatory models remain rare, explanatory generalizations of wide scope based on conserved genetic mechanisms appear more valued by developmental biologists than combined models of genetic and physical causes. A preference for generality fits with the standard genetic orientation of developmental biology as a discipline, but also has a wider application. Building integrated models is frequently the result of interdisciplinary efforts, where differences in explanatory standards are exposed; generalization within a discipline (with or without laws) is likely to take priority over integration across disciplines (at least in the short term). This implies that the tradeoff between generalization and integration identified for genetics and physics in developmental biology is broadly applicable, even if the particular idealization regarding temporal duration is not. Further analysis is required to enrich our understanding of explanatory practices and causal reasoning across different sciences.

Acknowledgement I am grateful to session participants at the 2015 European Philosophy of Science Association meeting in Düsseldorf and two anonymous referees for helpful feedback and suggestions that improved the final manuscript. I also benefited from comments on related material from audiences at Ottawa University and Yale University. The research and writing of this article was supported in part by a grant from the John Templeton Foundation (Integrating Generic and Genetic Explanations of Biological Phenomena; ID 46919). 


\section{References}

Brouzés, E., and E. Farge. 2004. Interplay of mechanical deformation and patterned gene expression in developing embryos. Current Opinion in Genetics \& Development 14: 367-374.

Craver, C.F., and L. Darden. 2013. In search of mechanisms: Discoveries across the life sciences. Chicago: University of Chicago Press.

Darden, L. 2006. Reasoning in biological discoveries: essays on mechanisms, interfield relations, and anomaly resolution. New York: Cambridge University Press.

Farge, E. 2003. Mechanical induction of twist in the Drosophila foregut/stomodeal primordium. Current Biology 13: 1365-1377.

Hopwood, N. 2005. Visual standards and disciplinary change: Normal plates, tables and stages in embryology. History of Science 43: 239-303.

Illari, P., and F. Russo. 2014. Causality: Philosophical theory meets scientific practice. New York: Oxford University Press.

Mill, J.S. 2006. A system of logic ratiocinative and inductive, being a connected view of the principles of evidence and the methods of scientific investigation. Indianapolis: Liberty Fund.

Miller, C.J., and L.A. Davidson. 2013. The interplay between cell signalling and mechanics in developmental processes. Nature Reviews Genetics 14: 733-744.

Mitchell, S.D. 2000. Dimensions of scientific law. Philosophy of Science 67: 242-265. - 2003. Biological complexity and integrative pluralism. New York: Cambridge University Press.

Savin, T., N.A. Kurpios, A.E. Shyer, P. Florescu, H. Liang, L. Mahadevan, and C. Tabin. 2011. On the growth and form of the gut. Nature 476: 57-62.

Sober, E. 1988. Apportioning causal responsibility. Journal of Philosophy 85: 303-318.

Varner, V.D., and C.M. Nelson. 2014. Cellular and physical mechanisms of branching morphogenesis. Development 141: 2750-2759.

Von Dassow, M., J. Strother, and L.A. Davidson. 2010. Surprisingly simple mechanical behavior of a complex embryonic tissue. PLoS ONE 5: e15359.

Waters, C.K. 2007. Causes that make a difference. Journal of Philosophy 104: 551-579.

Weisberg, M. 2007. Three kinds of idealization. Journal of Philosophy 104: 639-659.

Yashiro, K., H. Shiratori, and H. Hamada. 2007. Haemodynamics determined by a genetic programme govern asymmetric development of the aortic arch. Nature 450: 285-288.

Open Access This chapter is licensed under the terms of the Creative Commons Attribution 4.0 International License (http://creativecommons.org/licenses/by/4.0/), which permits use, sharing, adaptation, distribution and reproduction in any medium or format, as long as you give appropriate credit to the original author(s) and the source, provide a link to the Creative Commons license and indicate if changes were made.

The images or other third party material in this chapter are included in the chapter's Creative Commons license, unless indicated otherwise in a credit line to the material. If material is not included in the chapter's Creative Commons license and your intended use is not permitted by statutory regulation or exceeds the permitted use, you will need to obtain permission directly from the copyright holder. 